\title{
Modifications de l'activité de la protéine kinase C et de la phosphorylation de la lipocortine I dans les cultures de cellules thyroïdiennes porcines
}

\author{
F Antonicelli 1, B Omri 2, MF Breton 2, L Martiny 1, B Rothhut 3, \\ F Russo-Marie 3, B Lambert 1, M Pavlovic-Hournac ${ }^{2}$, B Haye ${ }^{*}$ \\ 1 Université de Reims, UFR sciences, laboratoire de biochimie, \\ BP 347, 51062 Reims Cedex; \\ 2 Unité INSERM 96, 78 rue du général Leclerc, 94275 Paris-Bicêtre; \\ ${ }^{3}$ Institut Pasteur, unité INSERM 285, 25 rue du docteur Roux, 75524 Paris cedex 15, France
}

(15 réunion du groupe Développement, INRA, Paris, 24-26 mai 1989)

\begin{abstract}
Résumé - En absence d'hormone, les cellules thyroïdiennes en culture se dédifférencient, perdent progressivement leur capacité de capter l'iodure et de l'organifier («cellules contrôles»). La présence de TSH $(0,1 \mathrm{mU} / \mathrm{ml})$ permet le maintien des fonctions différenciées en assurant une hyperorganification («cellules TSH»). L'addition de tétradécanoyl phorbol 13 acétate (TPA) entraîne une dédifférenciation rapide des cellules et des modifications importantes des différentes voies de signalisation. L'activité protéine kinase $\mathrm{C}$ (pKC), peu affectée dans les "cellules contrôles" et "cellules TSH», est fortement modifiée dans les cellules traitées par le TPA. Après une translocation rapide, l'activité pKC est "désensibilisée»" et la phosphorylation des substrats endogènes cytosoliques (35-38 kDa) disparaît. Parmi ces substrats de la pKC nous avons identifié la lipocortine I (LC I) (35kDa). Ces protéines antiphospholipasiques $A_{2}$ font partie de la famille des protéines fixant le $\mathrm{Ca}^{2+}$. Par électrophorèse monodimensionnelle (PAGE-SDS) et "électrotransfert" nous avons montré que la LCl est présente dans les fractions particulaires et cytosoliques des cellules. Par électrophorèse bidimensionnelle (PAGE-SDS et IEF) suivie d'un eélectrotransfert" nous avons montré que la LCl existe sous 2 formes, une phosphorylée et une non phosphorylée par la pKC. La phosphorylation de cette protéine déplace son pl de 6,9 à 6,6. Les modifications des différentes voies de signalisation, de l'activité de la pKC et de la phosphorylation de la $\mathrm{LCl}$ peuvent être, en partie, responsable de la dédifférenciation observée dans les cellules thyroïdiennes porcines cultivées en absence d'hormone ou en présence de TPA.
\end{abstract}

thyrocyte / dédifférenciation / ester de phorbol / protéine kinase C / lipocortine I

Summary - Modifications of protein kinase $C$ activity and lipocortin I phosphorylation in pig thyroid cultured cells. When cultured in the absence of thyreostimulin (TSH), thyroid cells lose some of their differentiated functions such as iodide transport and its incorporation into thyroglobulin. In the presence of TSH $(0.1 \mathrm{mU} / \mathrm{m} /$ ), these differentiated functions are preserved ( $\mathrm{TSH}$ cellsw). The addition of tetradecanoyl phorbol 13 acetate (TPA) inhibits some differentiated functions of the cells and provokes important modifications of bio-signalling pathways. The protein kinase $C$ (pKC) activity, unchanged in "control" and "TSH cells", was dramatically modified in TPA treated cells. After translocation, the pKC activity was down-regulated and the phosphorylation of its endogenous sub-

* Correspondance et tirés à part 
strates (35-38 kDa) disappeared. Among these substrates, we identified the lipocortin I (LC I) (35 $k D a)$, a phospholipase $\mathrm{A}_{2}$ inhibitory protein related to the $\mathrm{Ca}^{2+}$ binding protein family. By monodimensional electrophoresis (PAGE-SDS) and western-blot, we evidenced the presence of $L C l$ in cytosols and particulate extracts. By 2 dimensional electrophoresis (PAGE-SDS and IEF) and western-blot we identified a phosphorylated and unphosphorylated LCl protein. The phosphorylation of LCl by pKC decreased its isoelectric point from 6.9-6.6. The modifications of pKC activity and LCl phosphorylation and the changes in the bio-signalling pathways can partly account for the loss of differentiation observed in control or TPA treated cells.

thyrocyte / differentlation / phorbol ester / protein kInase C / Ilpocortin I

\section{INTRODUCTION}

II est généralement admis actuellement que les cellules thyroïdiennes en culture se dédifférencient quand elles sont maintenues sur milieux dépourvus de tous régulateurs (Kerkof et al, 1964; Fayet et al, 1971; Lissitzky et al, 1971; Mauchamp et al, 1980; Roger et Dumont, 1984; Errick et al, 1985). Elles ne peuvent être maintenues à l'état différencié que quand elles sont cultivées en présence de la TSH ou de la forskoline (activateur direct de l'adénylate cyclase) (Kerkof et al, 1964; Fayet et al, 1971; Mauchamp et al, 1980; Roger et Dumont, 1984; Errick et al, 1985). En ce qui concerne le tétradécanoyl phorbol 13 acétate (TPA), ester de phorbol ayant une action promotrice de tumeurs, ses effets dépendent de la durée de son contact avec les cellules. A très court terme, $1<30$ min), le TPA modifie la production de I'AMPc induite par la TSH ou la forskoline (Bachrach et al, 1985; Haye et al, 1985a; Omri et al, 1988). Dans ces mêmes conditions, il stimule également lincorporation de l'iodure dans les protéines (Levasseur et al, 1985). Son effet à long terme se manifeste par une dédifférenciation accélérée des cellules en culture.

Étant donné que le TPA agit principalement par l'activation de la protéine kinase $\mathrm{C}$, qui est son récepteur, nous avons mesuré l'activité de cette enzyme et analysé la phosphorylation de ses substrats spécifiques dans les cellules exposées au TPA pendant des temps variables. Parmi les substrats de la pKC nous avons identifié la lipocortine de type I dont le poids moléculaire est de $35 \mathrm{kDa}$.

Nous avons également évalué les taux des médiateurs intracellulaires des 2 voies de régulation de la cellule thyroïdienne (AMP cyclique et phospho-inositides) et comparé l'expression de la principale fonction thyroïdienne (l'incorporation de l'iodure dans les protéines) dans les cellules différenciées et en cours de dédifférenciation.

\section{MATÉRIEL ET MÉTHODES}

\section{Préparation, culture et lavage des cellules thyroïdiennes}

Les cellules thyroïdiennes ont été isolées à partir des glandes de porc, par la technique de trypsinisation discontinue (Mauchamp et al, 1980).

La préparation, la culture et le lavage des cellules thyroïdiennes ont été réalisés d'après Haye et al $(1985 a, b)$.

\section{Préparation des fractions cytosoliques et particulaires}

La préparation des fractions cytosoliques et particulaires à partir de cultures thyroïdiennes, ainsi que la mesure de l'activité pKC et la phosphory- 
lation des protéines endogènes ont été conduites d'après la technique décrite par Omri et al (1988).

\section{Phosphorylation des protéines endogènes in vitro}

\section{Électrophorèse monodimensionnelle}

Les protéines phosphorylées ont été analysées par électrophorèse en milieu dénaturant (SDS), sur des gels de polyacrylamides (gradient 5$15 \%$ ) suivant la technique décrite par Laemmli (1970). Après la migration, la lipocortine I a été identifiée par électrotransfert suivant la technique de Rothhut et al (1987). Les protéines phosphorylées ont été localisées par autoradiographie.

\section{Électrophorèse bidimensionnelle}

La séparation des protéines par électrophorèse bidimensionnelle a été réalisée suivant la technique décrite par O'Farrel (1975).

Détermination de l'AMP cyclique, étude du renouvellement des phospholipides, mesure de l'organification de l'iodure

Tous ces paramètres ont été mesurés ou testés selon les méthodes décrites par Haye et al (1985b).

L'incorporation de ${ }^{32} \mathrm{P}$ dans les phosphoinositides (PI) sur l'incorporation de 32P dans la phosphatidylcholine (PC) est utilisée comme index (PI/PC) (Gérard et al, 1982).

\section{Autres méthodes utilisées}

Les protéines ont été mesurées par la méthode de Lowry et al (1951); le contenu en ADN a été déterminé selon la méthode de Groyer et Robel (1980) sur une aliquote de $100 \mu \mathrm{l}$ de l'homogénat.

\section{Expression des résultats, analyse statistique}

Les différences statistiques entre les essais ont été calculées en utilisant le test $t$ de Student. Les expériences ont été reproduites au minimum 3 fois avec les mêmes résultats. Le résultat d'une expérience significative est présenté. Une valeur de $P<0,01$ est considérée comme significative dans la comparaison des différentes séries.

\section{RÉSULTATS}

Métabolisme des cellules thyrö̈diennes en culture apres un traitement chronique de deux jours par la TSH $(0,1 \mathrm{mU} / \mathrm{ml})$ ou le TPA $\left(10^{-7} \mathrm{~mol} / \mathrm{l}\right)$

\section{Sur l'organification de l'iodure}

Dans les cellules cultivées pendant 2 j en présence de $\mathrm{TSH}$, l'incorporation de l'iodure dans les protéines est très fortement augmentée par rapport au contrôle, alors que dans les cellules traitées durant 2 j par le TPA, elle est inhibée (fig 1).

\section{Rapports PI/PC}

Dans les "cellules TSH", le rapport PI/PC augmente fortement pendant la culture, alors que dans les "cellules TPA" il diminue d'une façon spectaculaire (fig 1). Ceci est dû aux modifications des renouvellements à la fois du Pl et de PC, qui sont très différents dans les "cellules TSH» et «TPA» (fig 2).

\section{Production des prostaglandines $E_{2}$}

Dans les "cellules TSH", la production des $\mathrm{PGE}_{2}$ est très inhibée par rapport à celle observée dans les "cellules contrôles". 

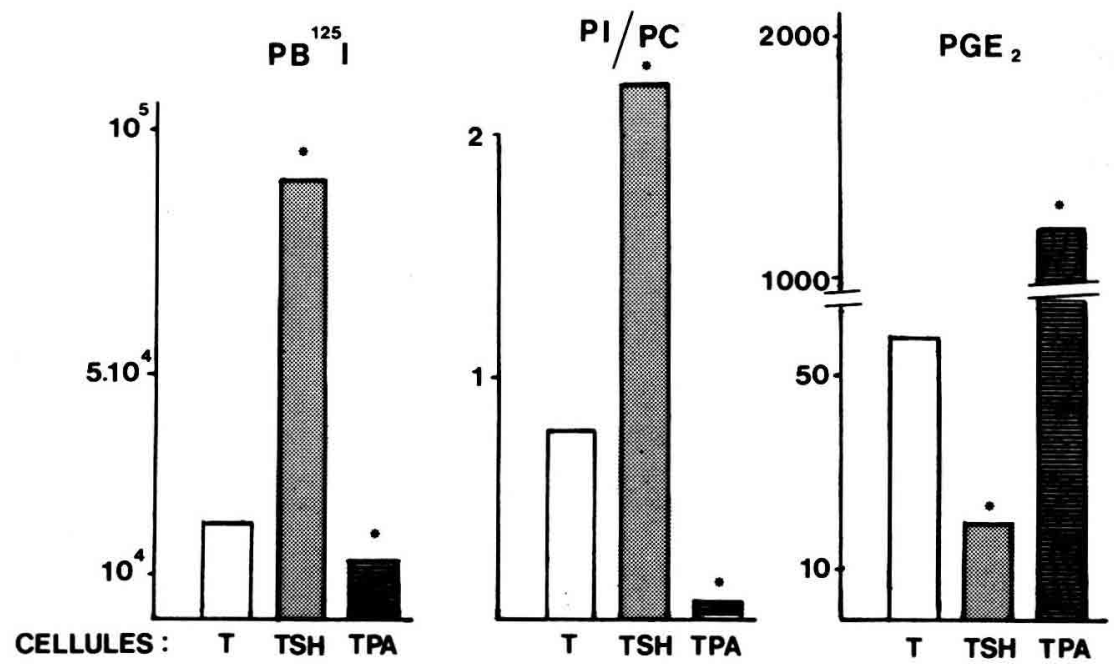

Fig 1. Après 2 j de culture en absence d'agoniste (cellules contrôles «T») ou en présence de TSH $(0,1 \mathrm{mU} / \mathrm{ml})$ ou TPA $\left(10^{-7} \mathrm{~mol} / \mathrm{l}\right)$, les cellules sont lavées et incubées pendant $45 \mathrm{~min}$ en présence de $1 \mu \mathrm{Ci}$ 125l-Na pour la mesure de l'organification de l'iodure (PB 125l), pendant $2 \mathrm{~h}$ en présence de $32 \mathrm{P}$ orphophosphate pour la mesure du renouvellement des phospholipides $(\mathrm{PI}=$ phospho-inositides, $\mathrm{PC}=$ phosphatidylcholine), pendant $5 \mathrm{~h}$ pour la mesure de la production de $\mathrm{PGE}_{2}$ (dosage radioimmunologique). Analyse statistique : $P<0,001$ par rapport au témoin correspondant.

Par contre, les cellules cultivées en présence du TPA ont une production très élevée de $\mathrm{PGE}_{2}$ (fig 1).

\section{Production de I'AMPC}

Contrairement aux médiateurs de la voie phospholipidique, le taux basal de l'AMPc varie très peu au cours de la culture dans les 3 types cellulaires étudiés (contrôle, TSH, TPA) (résultats non montrés).

\section{Activité de la protéine kinase $C(p K C)$}

Dans les glandes thyroïdes de porc, ainsi que dans les cellules thyroïdiennes en culture, l'activité prédominante des protéines kinases et celle de la pKC. Au cours de la culture son activité n'est significativement modifiée ni dans les "cellules contrôles» ni dans celles cultivées en présence de TSH. Le TPA, par contre, provoque de très importantes modifications de son activité (fig 3). A court terme, il induit une translocation très rapide de l'enzyme, de la fraction soluble vers la fraction particulaire (fig 4). A partir de 5-10 min (maximum $30 \mathrm{~min}$ ) l'activité de la pKC disparaît presque complètement (plus de 95\%) de la fraction soluble. Cette désensibilisation de la pKC de la fraction soluble est maintenue pendant toute la période de culture des cellules en présence du TPA. Dans la fraction particulaire, l'enzyme transloquée atteint le maximum d'activité entre 15-30 min, puis son activité commence à décroître progressivement. Après 2 j de culture l'activité pKC particulaire est négligeable. 


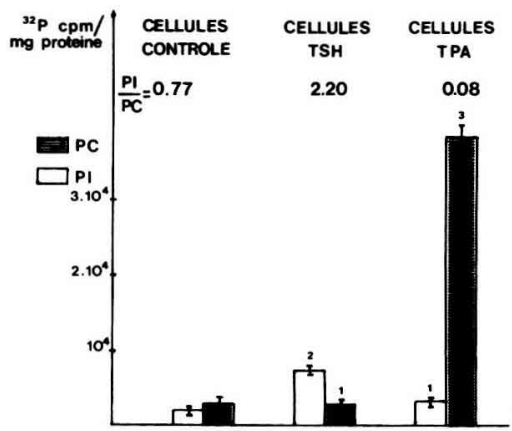

Fig 2. Après $2 \mathrm{j}$ de culture en absence d'agoniste (cellules contrôles $\alpha T \omega$ ) ou en présence de TSH $(0,1 \mathrm{mU} / \mathrm{ml})$ ou TPA $\left(10^{-7} \mathrm{~mol} / \mathrm{l}\right)$, les cellules sont lavées et incubées pendant $2 \mathrm{~h}$ en présence de ${ }^{32} \mathrm{P}$-orphophosphate pour la mesure du renouvellement des phospholipides (PI $=$ phospho-inositides, $\mathrm{PC}=$ phosphatidylcholine). Analyse statistique : 1) Non significativement différent du phospholipide correspondant des "cellules contrôles». 2) $P<0,01$ par rapport au phospholipide correspondant des "cellules contrôles». 3) $P<0,001$ par rapport au phospholipide correspondant des "cellules contrôles».
La désensibilisation de la pKC dans les cellules cultivées se répercute sur la phosphorylation de ses substrats spécifiques.

\section{Substrats de pKC}

Après $2 \mathrm{j}$ de culture, en absence ou en présence de TSH $(0,1 \mathrm{mU} / \mathrm{ml})$ ou TPA $\left(10^{-7} \mathrm{~mol} / \mathrm{l}\right)$, les substrats de la pKC sont mis en evidence par phosphorylation in vitro des protéines de la fraction soluble ou particulaire avec l'ATP- $\gamma$-32P. En présence de calcium et de phospholipides (CaPL), on constate que les substrats majeurs de la pKC, aussi bien dans la fraction soluble (fig 5) que particulaire (non montré), sont les bandes protéiques ayant un poids moléculaire entre 35 et $38 \mathrm{kDa}$. La phosphorylation de ces protéines est absente des cellules dans lesquelles la pKC est désensibilisée par le TPA pendant $2 \mathrm{j}$.

\section{Identification de la lipocortine I ( $L C$ I) comme un des substrats de la pKC}

Récemment (Antonicelli et al, 1988), nous avons purifié et caractérisé dans la thy-
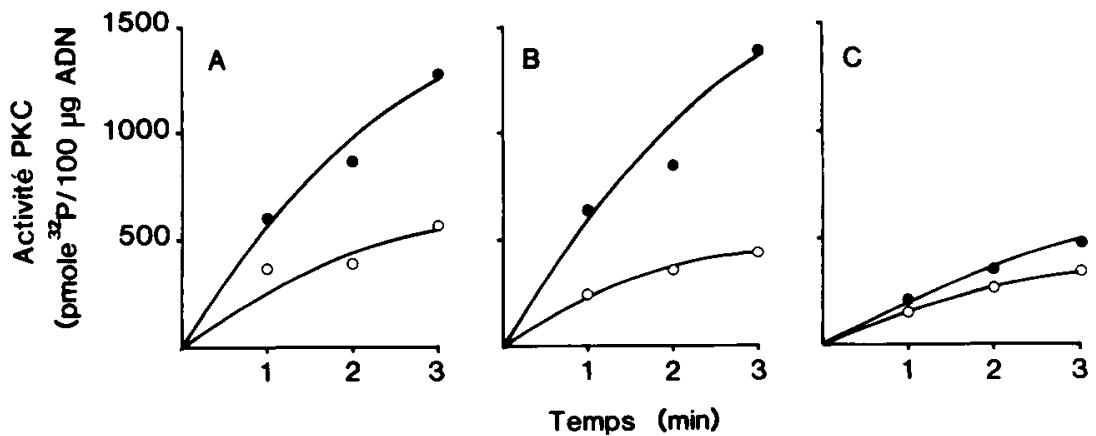

Fig 3. L'activité protéine kinase $C$ a été évaluée en absence $\left(\mathrm{O}_{-}-\mathrm{O}\right)$ ou en présence $(-\longrightarrow)$ de $\mathrm{Ca}^{2+}$ et de phospholipides. A : "Cellules contrôles". B : “Cellules TSH». C : "Cellules TPA". 


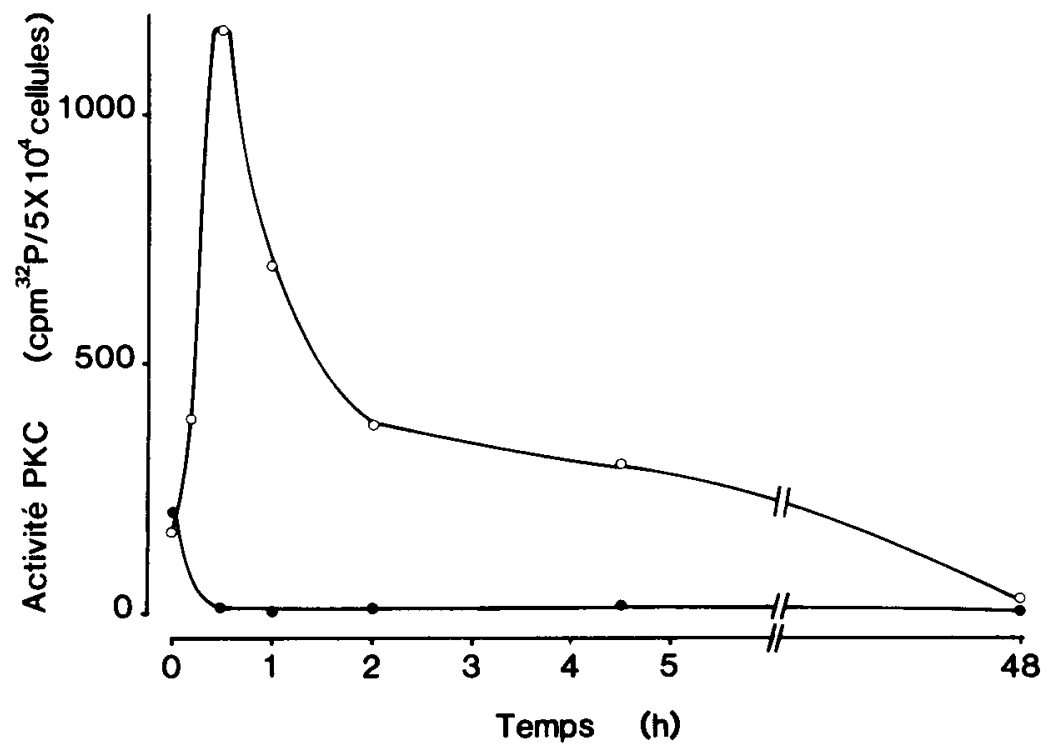

Fig 4. L'activité pKC a été déterminée dans le cytosol (-) ou dans les particules $\left(\mathrm{O}_{-}^{-} \mathrm{O}\right)$ en présence de $\mathrm{Ca}^{2+}$ et de phospholipides.

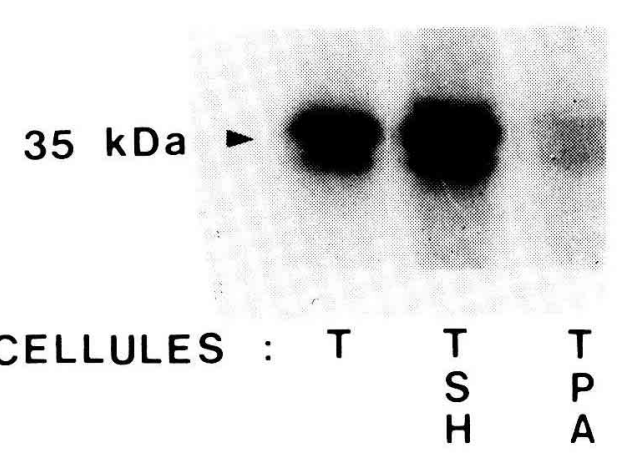

Fig 5. Après les $2 \mathrm{j}$ de culture et la préparation des fractions solubles des différents lots cellulaires, "T", "TSH", "TPA", la phosphorylation des protéines de la fraction soluble par la pKC a été déterminée en présence de $\mathrm{Ca}^{2+}$ et de phospholipides. Après incubation de 2 min en présence d'ATP- $\gamma$-32P, les protéines phosphorylées sont analysées d'après la méthode de Laemmli (1970) et localisées par autoradiographie. roïde une protéine antiphospholipasique $A_{2}$ de type lipocortine appelée endonexine (32 kDa). Parallèlement, nous avons mis en évidence une autre lipocortine de type I (35 $\mathrm{kDa})$ et sa protéine core (33 kDa). Comme ces protéines ont des masses molaires analogues à celles des substrats de la pKC, nous avons étudié leur phosphorylation par la pKC. Après phosphorylation par la pKC et électrophorèse en condition dénaturante suivie d'un électrotransfert, et en utilisant l'anticorps antilipocortine I, on constate la présence de la lipocortine de $35 \mathrm{kDa}$ dans les fractions soluble et particulaire provenant des "cellules TSH" et "TPA" (fig 6). Dans la fraction particulaire de ces 2 lots cellulaires, on retrouve également la protéine core de 33 kDa dont la présence est plus importante dans les particules des "cellules TPA" que dans les "cellules TSH". 
CELLULES TSH Cytosol Particules Cytosol Particules

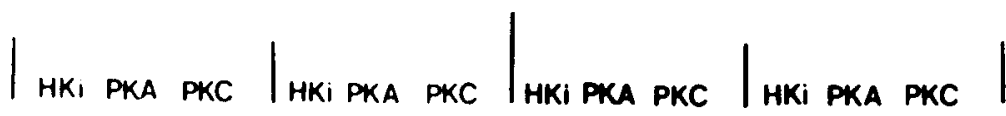

\section{$67-$}

$43-$

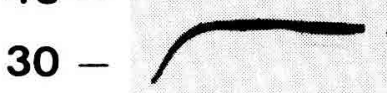

Anti

\section{LC I}
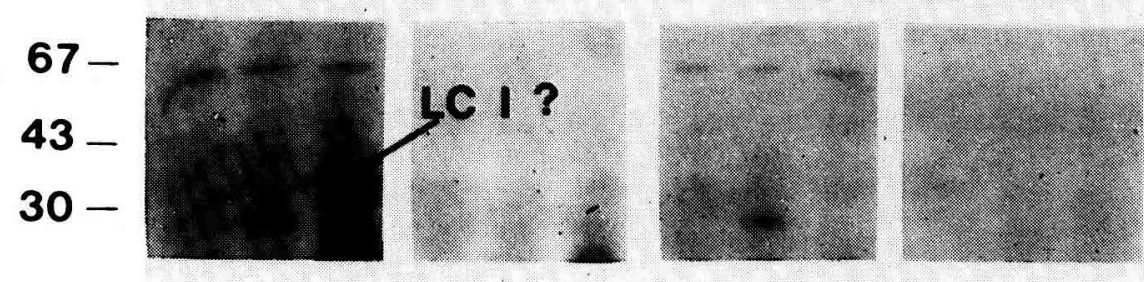

\section{Autoradiographie}

Fig 6. Électrophorèse monodimensionnelle, électrotransfert et autoradiographie des protéines solubles et particulaires après phosphorylation par la pKC. HKi : activité basale en absence de $\mathrm{Ca}^{2+}$ et de phospholipides. pKA : phosphorylation par la protéine kinase A. pKC : phosphorylation par la protéine kinase $\mathrm{C}$ en présence de $\mathrm{Ca}^{2+}$ et de phospholipides. Les conditions expérimentales sont les mêmes que pour la figure 5 sauf qu'un électrotransfert a précédé l'autoradiographie.

Après autoradiographie, on constate que la bande de $35 \mathrm{kDa}$, substrat spécifique de la pKC dans les cellules en culture, est superposable à la bande révélée par l'anticorps antilipocortine.
Afin de mieux préciser la nature des lipocortines phosphorylées par la pKC, une électrophorèse bidimensionnelle suivie d'un électrotransfert a été réalisée sur des extraits protéiques cytosoliques des 
"cellules TSH" uniquement, puisque dans les "cellules TPA" les substrats endogènes ne peuvent pas être phosphorylés, la pKC étant désensibilisée. De plus, 4 différents anticorps antilipocortines ont été utilisés : anti $32 \mathrm{kDa}$ (LC V); anti $35 \mathrm{kDa}$ (LC I); anti $36 \mathrm{kDa}$ (LC II) et anti $70 \mathrm{kDa}$ (LC VI).

La coloration de l'électrotransfert par la $\mathrm{DAB}$ a révélé la présence des 4 lipocortines (LC I, LC II, LC V, LC VI) dans le cytosol des "cellules TSH» (fig 7, haut). II faut noter que la lipocortine I (LC I) est présente sous forme d'une tache majeure dont le pl est de 6,9 et d'une tache mineure ayant un pl de 6,6. Cette dernière n'est présente que lorsque la pKC est activée.

La superposition de l'électrotransfert et de l'autoradiographie (fig 7, bas) a permis de définir les lipocortines phosphorylées par la pKC dans la thyroïde :

- les lipocortines $I I, V$ et $V I$ ne sont pas phosphorylées par la pKC (CaPL);

- la lipocortine 1 existe sous 2 formes, une phosphorylée et une non phosphorylée. La phosphorylation déplace apparemment son point isoélectrique de 6,9 à 6,6 .

\section{DISCUSSION}

Le traitement pendant $2 \mathrm{j}$ par le TPA $\left(10^{-7}\right.$ mol/l) des cellules thyroïdiennes en culture provoque des modifications importantes de leur métabolisme, de leur stimulation par l'hormone, et de leur morphologie (Haye et al, 1985a; Omri et al, 1988). Le niveau basal de la production de cAMP est peu affecté, mais sa stimulation par l'hormone est fortement inhibée. L'incorporation de $32 \mathrm{P}$ orthophosphate dans les phospholipides est réorientée vers la phosphatidylcholine, ce qui se traduit par une modification importante du rapport PI/ PC observé dans les "cellules contrôles" ou les "cellules TSH". L'organification de l'iodure, élevée dans les "cellules TSH", est inhibée dans les cellules traitées par le TPA. La synthèse de la thyroglobuline est, elle aussi, inhibée alors que celle du collagène de type IV est augmentée (G Bellon et al, non publié). De plus, les cellules cultivées en suspension, qui ne se divisent pas, prennent sous l'action du TPA l'aspect de cellules fusiformes et adhèrent progressivement au support.

Après une translocation rapide de la pKC par le TPA, les cellules thyroïdiennes perdent progressivement cette activité. Parmi les substrats endogènes de la pKC, nous avons identifié la lipocortine I (35 kDa) dans les "cellules TSH". Dans les "cellules TPA" ce substrat, toujours présent, n'est plus phosphorylé et ceci se traduit par une dérégulation de l'activité phospholipasique $A_{2}$ qui se répercute sur la production des $P G E_{2}$. Dans les "cellules contrôles" et les "cellules TPA", dédifférenciées ou en cours de dédifférenciation, l'activité phospholipasique $A_{2}$ est élevée. Elle est faible dans les "cellules TSH». Dans notre système il paraît y avoir une bonne corrélation entre la phosphorylation de la lipocortine I et l'activité de la phospholipasique $A_{2}$, l'absence de phosphorylation est observée dans les celules TPA où l'activité phospholipasique $A_{2}$ est élevée. Ceci est contradictoire avec le modèle précédemment décrit par Hirata (1981). La régulation de l'activité des lipo-cortines paraît plus complexe qu'un simple mécanisme de phosphorylation-déphosphorylation. Un contrôle peut intervenir au niveau de l'expression, de la protéolyse ménagée, de la fixation du $\mathrm{Ca}^{2+}$ et des phospholipides, de la translocation, de la phosphorylation par d'autres protéines kinases (tyrosine kinase) (Rainteau et al, 1988; Davidson et al, 1987; Huang et al, 1987; Sato et al, 1988; Schlaepfer et Haigler, 1987). 

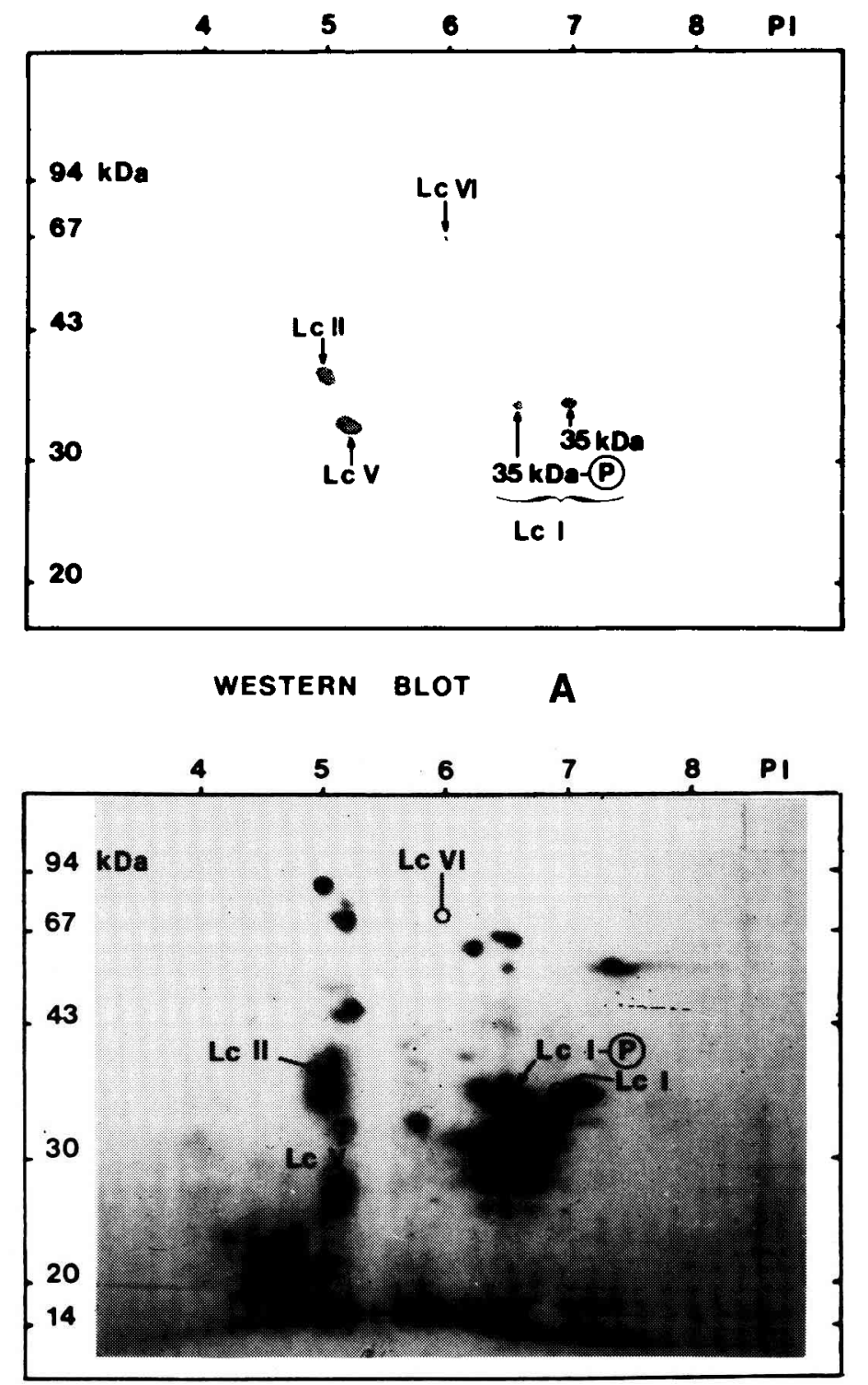

\section{AUTORADIOGRAPHIE B}

Fig 7. Électrophorèse bidimensionnelle, électrotransfert et autoradiographie des protéines solubles des «cellules TSH" après phosphorylation par la pKC. Les conditions expérimentales sont les mêmes que pour les figures 5 et 6 sauf que l'expérience a été arrêtée selon la méthode décrite par O'Farrell (1975). Quatre anticorps ont été testés successivement après l'électrotransfert et avant l'autoradiographie. 
Par ailleurs, il n'est pas exclu que ces protéines aient d'autres fonctions biologiques dont la nature, l'importance et la localisation restent à démontrer (pour une synthèse voir Crompton et al, 1988).

\section{REMERCIEMENTS}

Nous remercions Madame $O$ Legue pour son excellente collaboration technique et Madame Subtil qui a réalisé le manuscrit. Nous remercions également le NIH (Bethesda) et la Sobevir (Rethel, France) qui ont donné respectivement la TSH et les thyroïdes de porc, ainsi que le Docteur J Browning (Biogen, USA) qui nous a fourni gracieusement certains anticorps. $\mathrm{Ce}$ travail a été en partie réalisé grâce à des subventions de la Fondation de la recherche médicale et de l'Association pour la recherche sur le cancer et grâce à un contrat avec l'INSERM (CRE $n^{\circ}$ 874009).

\section{RÉFÉRENCES}

Antonicelli $F$, Rothhut $B$, Martiny L, Aguie-Aguie G, Lambert B, Bellon G, Russo-Marie F, Jacquemin $C$, Haye $B$ (1988) Purification and characterization of phospholipase $\mathrm{A}_{2}$ inhibitory proteins from pig thyroid gland. FEBS Lett 235, 252-256

Bachrach L, Eggo MC, Mak WW, Burrow GN (1985) Phorbol esters stimulate growth and inhibit differentiation in cultured thyroid cells. Endocrinology 116, 1603-1609

Crompton MR, Moss SE, Crumpton MJ (1988) Diversity in the lipocortin/calpactin family. Cell 55, 1-3

Davidson FF, Dennist EA, Powell M, Glenney JR (1987) Inhibition of phospholipase $A_{2}$ by "lipocortin" and calpactins. J Biol Chem 262, 1698-1705

Errick JE, Eggo MC, Burrow GN (1985) Epidermal growth factor inhibits thyrotropinmediated synthesis of tissue-specific proteins in cultured ovine thyroid cells. Mol Cell Endocrinol 43, 51-59
Fayet G, Michel-Bechet M, Lissitzky S (1971) Thyrotropin-induced aggregation and reorganization into follicles of isolated porcine thyroid cells in culture. Ultrastructural studies. Eur J Biochem 24, 100-111

Gérard C, Haye B, Jacquemin C, Mauchamp J (1982) Chronic and acute effects of thyrotropin on phosphatidylinositol turnover in cultured porcine thyroid cells. Biochim Biophys Acta 710, 359-369

Groyer A, Robel P (1980) DNA measurement by mithramycin fluorescence in chromatin solubilized by heparin. Anal Biochem 106, 262268

Haye B, Aublin JL, Champion S, Lambert B, Jacquemin C (1985a) Tetradecanoyl phorbol13-acetate counteracts the responsiveness of cultured thyroid celis to thyrotropin. Biochem Pharmacol 34, 3795-3802

Haye B, Aublin JL, Champion S, Lambert B, Jacquemin C (1985b) Chronic and acute effects of forskolin on isolated thyroid cell metabolism. Mol Cell Endocrinol 43, 41-50

Hirata $F$ (1981) The regulation of lipomodulin, a phospholipase inhibitory protein, in rabbit neutrophils by phosphorylation. $J$ Biol Chem 256, 7730-7733

Huang KS, McGray P, Mattaliano RJ, Burne C, Pingchang Chow E, Sinclair LK, Pepinski RB (1987) Purification and characterization of proteolytic fragments of lipocortin I that inhibit phospholipase $A_{2}$. J Biol Chem 262, 7639 7645

Kerkof PR, Tong PJ, Chaikoff IL (1964) In vitro effects of thyrotropic hormone. I. On the pattern of organization of monolayer cultures of isolated sheep thyroid gland cells. Endocrinology $74,170-179$

Laemmli UK (1970) Cleavage of structural proteins during the assembly of the head of bacteriophage T4. Nature 227, 680-685

Levasseur S, Morgan L, Friedman Y, Burke $G$ (1985) Forskolin and 12-0-Tetradecanoylphorbol-13-acetale mimic thyrotropinstimulated protein iodination in mouse thyroid. Biochem Biophys Res Comm 128, 858863

Lissitzky S, Fayet G, Giraud A, Verrier B, Torresani $J$ (1971) Thyrotropin-induced aggregation and reoganization into follicles of isolated porcine thyroid cells. Mechanism of action of 
thyrotropin and metabolic properties. Eur $J$ Biochem 24, 88-99

Lowry OH, Rosebrough NJ, Farr AL, Randall RJ (1951) Protein measurement with the folin phenol reagent. $J$ Biol Chem 193, 265-275

Mauchamp J, Chambard M, Gabrion J, Pelassy C (1980) Polarisation morphologique et fonctionnelle d'un épithélium simple en culture : le modèle thyroïdien. CR Soc Biol 174, 241 256

O'Farrell PH (1975) High resolution twodimensional electrophoresis of proteins. $J$ Biol Chem 250, 4007-4021

Omri B, Breton MF, Haye B, Jacquemin C, Pavlovic-Hournac M (1988) Phorbol ester prevents the thyroid-stimulating-hormoneinduced but not the forskolin-induced decrease of CAMP-dependent protein kinase activity in thyroid cell cultures. Eur J Biochem $175,125-133$

Rainteau DP, Weinman SJ, Kabaktchis CAM, Smith VL, Kaetzel MA, Dedman JR, Weinman JS (1988) The expression of the 35- and
$67 \mathrm{kDa}$ calcimedins is dependent on thyroid hormone. J Biol Chem 263, 12844-12848

Roger PP, Dumont JE (1984) Factors controlling proliferation and differentiation of canine thyroid cells cultured in reduced serum conditions: effects of thyrotropin, cyclic AMP and growth factors. Mol Cell Endocrinol 36, 79-93

Rothhut B, Comera C, Prieur B, Errasfa M, Minassian G, Russo-Marie F (1987) Purification and characterization of a 32-kDa phospholipase $A_{2}$ inhibitory protein (lipocortin) from human peripheral blood mononuclear cells. FEBS Lett 219, 169-175

Sata EF, Okimasu E, Takahashi R, Miyahara M, Matsuno T, Utsumi K (1988) Lipocortin-like $33 \mathrm{kDa}$ protein of guinea pig neutrophil. Its distribution and stimulation-dependent translocation detected by monoclonal anti-33 $\mathrm{kDa}$ protein antibody. Cell Struct Funct 13, 89-96

Schlaepfer DD, Haigler HT (1987) Characterization of $\mathrm{Ca}^{2+}$-dependent phospholipid binding and phosphorylation of lipocortin I. J Biol Chem 262, 6931-6937 